\title{
NEUTRINOS AND LARGE SCALE STRUCTURES
}

\author{
P.R. Dhungel*, S.K. Sharma** and U. Khanal* \\ *Central Department of Physics, Tribhuvan University, Kirtipur, Kathmandu, Nepal. \\ **BPKM Planetarium, Observatory \& Science Museum DB, Ministry of Env., S\&T, Kathmandu, Nepal.
}

\begin{abstract}
We have summarized the neutrino masses detected/predicted from different sources so far and calculated the Jeans masses of the neutrino structures for these values of neutrino masses. The size of the pure neutrino structures has come to be bigger than the superclusters and great attractor formed around 'recombination epoch'.
\end{abstract}

Key Words: Neutrino; Large-scale structures; Jeans mass; Relic neutrino; Supercluster; Recombination epoch.

\section{INTRODUCTION}

Neutrinos are the second most abundant particles in the universe, with a number density comparable to that of relic photons. Therefore, the relic neutrino sea influences various cosmological situations, playing an important role that has been discussed in many papers, both to solve some cosmological problems and to put bounds on non-standard neutrino properties. In this paper, we have summarized the consecutive development of our knowledge of the upper limit of neutrino mass and hence its implication in the large-scale structures. The latter part has been done by calculating the Jeans mass for the neutrino masses known to us at various time by various sources.

Several years before neutrinos had even been experimentally detected, Alpher, Folin \& Hermann $\left[{ }^{1},{ }^{2}\right]$ noted that they would have been in thermal equilibrium in the early universe "...... through interactions with mesons” at temperatures above 5 $\mathrm{MeV}$; below this temperature the neutrinos “....... freeze-in and continue to expand and cool adiabatically as would a pure radiation gas". These authors also observed that the subsequent annihilation of $\mathrm{e}^{+} \mathrm{e}^{-}$pairs would heat the photons but not the decoupled neutrinos.

Zel'dovich[ $\left.{ }^{3}\right]$ and Chiu[ $\left.{ }^{4}\right]$ had made the conclusion that the relic neutrinos, although nearly as numerous as the blackbody photons, cannot make an important contribution to the cosmological energy density since they are probably massless. But some years earlier Pontecoro \& Smorodinski[5] had discussed the bounds set on the cosmological energy density of $\mathrm{MeV}$ energy neutrinos using data from the ReinesCowan and Davis experiments.
Several years later, Gershtein \& Zel'dovich[ $\left.{ }^{6}\right]$ made the connection that if relic neutrinos are massive, then a bound on the mass follows from simply requiring that $m_{v} n_{v}<\rho_{m}$. They derived $\rho_{\mathrm{m}}<2 \times 10^{-28} \mathrm{gm} \mathrm{cm}^{-3}$ and inferred that $m_{v_{e}}, m_{v_{\mu}}<400$ $\mathrm{eV}$ for a present photon temperature of $3 \mathrm{~K}$. A better bound of $m_{v_{\mu}}<130 \mathrm{eV}$ was quoted by Marx \& Szalay[ $\left.{ }^{7}\right]$ who numerically integrated the cosmological Friedmann equation from $v_{\mu}$ decoupling down to the present epoch, subject to the condition $\mathrm{t}_{\mathrm{o}}>4.5 \mathrm{Gyr}$. Independently Cowsik \& McClleland $\left[{ }^{8}\right]$ used direct limits on $\Omega_{\mathrm{m}}$ and h to obtain an even more restrictive bound of $m_{v}<8 \mathrm{eV}$, assuming that $m_{v}=m_{v_{e}}=m_{v_{\mu}}$; however they too assumed that $\mathrm{T}_{v}=\mathrm{T}$ and that right handed states were fully populated. Thus we arrive at the modern version of the 'Gershtein-Zel'dovich bound' [']: the conservative limits $\mathrm{t}_{\mathrm{o}}>10 \mathrm{Gyr}$ and $\mathrm{h}>0.4$ imply that $\Omega_{\mathrm{m}} \mathrm{h}^{2}<1$; combining this with the relic neutrino number density gives $\Omega_{v} h^{2}=\sum_{i}\left(\frac{m_{v_{i}}}{93 \mathrm{eV}}\right)\left(\frac{g_{v_{i}}}{2}\right)<1$. Together with the Hubble Key Project determination of $h=0.72 \pm 0.08$, this implies that the sum of all neutrino masses cannot exceed about $15 \mathrm{eV}$. Although this has historically been the most restrictive constraint on neutrino masses, it is no longer competitive with direct laboratory bound on the neutrino mass and much more precise results from recent Wilkinson Microwave Anisotropy Probe (WMAP).

Before WMAP data, the best bound on $\Sigma \mathrm{m}_{v}$ was obtained using the power spectrum of galaxy clustering from the Two Degree Field Galaxy Redshift Survey (2dFGRS) and adding other independent cosmological constraints through priors on the other parameters $\left[{ }^{10}\right]$. The upper limit found was $\Sigma \mathrm{m}_{v}$ $<2.2 \mathrm{eV}$ (95\% CL). The upper bound on neutrino masses reported by the WMAP collaborators[ $\left[{ }^{11}\right]$, using the first-year

Author for Correspondence: Prof. Dr. Udayaraj Khanal, Central Department of Physics, Tribhuvan University, Kirtipur, Kathmandu, Nepal. 
WMAP data, Cosmic Microwave Background (CMB) data from other experiment (Arcminute Cosmology Bolometer Array Receiver (ACBAR) and Cosmic Background Imager (CBI)), the LSS data from 2dFGRS and the matter power spectrum on small scales inferred from the Lyman á forest was $\Sigma \mathrm{m}_{v}<0.7 \mathrm{eV}$ (95\% CL). The 5 year-WMAP $\left.{ }^{12}\right]$ data combined with the distance information from Baryon Acoustic Oscillations (BAO) and Supernova (SN), the limit on the neutrino mass has been obtained to be $\Sigma \mathrm{m}_{v}<0.61 \mathrm{eV}$ (95\% CL), which is better than the 5yr-WMAP only limit by a factor of 2: $\Sigma \mathrm{m}_{v}<1.3 \mathrm{eV}$.

The pioneering calculation by $\mathrm{W}$. Hu et al.[13] found that the combination of future CMB and Large Scale Structure (LSS) data, and in particular the Sloan Digital Sky Survey (SDSS) will push the bound on $\Sigma \mathrm{m}_{v}$ to approximately $0.3 \mathrm{eV}$. More recently, Hannestad $\left[{ }^{14}\right]$ has updated the forecast analysis and concluded that the PLANCK CMB experiment combined with SDSS data could measure a neutrino mass of $0.12 \mathrm{eV}$ at $95 \%$ CL. With an order of magnitude larger survey volume than SDSS, the limit could be pushed down to $0.03-0.05 \mathrm{eV}\left[{ }^{15}\right]$.

When we $\left[{ }^{16}\right]$ calculated the Jeans mass of large-scale structures of neutrinos and plotted its variation with temperatures, it was found to be peaked at different temperatures to different peak values for different combinations of parameters and the averaging processes. The lowest peak which occurred at the earliest was at $\mathrm{x}=\mathrm{m}_{\mathrm{v}} \mathrm{c}^{2} / \mathrm{k}_{\mathrm{B}} \mathrm{T}=1.9$. In this paper, we will see the effect of the improvements in the neutrino mass limits on the Jeans mass and hence on the sizes of the of the neutrino structures in the large-scales.

\section{JEANS MASS OF NEUTRINO STRUCTURES:}

As calculated in our paper [16], the number density distribution of neutrinos in momentum space is given by $d n=\frac{g}{2 \pi^{2}}\left(\frac{m}{x}\right)^{3} \frac{y^{2}}{e^{y}+1} d y$, where $\mathrm{m}$ is rest mass of neutrino, $\mathrm{X}=\mathrm{m}_{v} \mathrm{C}^{2} /$ $\mathrm{k}_{\mathrm{B}} \mathrm{T}, \mathrm{y}=\mathrm{pc} / \mathrm{k}_{\mathrm{B}} \mathrm{T}$, $\mathrm{g}$ is the spin degeneracy number. Integrating this over 0 to $\infty$ gives the number density $n=\frac{g}{2 \pi^{2}} \Gamma(3) \eta(3)\left(\frac{m}{x}\right)^{3}$ where $\Gamma(3)$ and $\eta(3)$ are the gamma and Riemann eta functions respectively. Thus we can write down the expectation value of any regular function $\mathrm{f}(\mathrm{y})$ as $\langle\mathrm{f}(\mathrm{y})\rangle=\frac{1}{\Gamma(3) \eta(3)} \int d y \frac{y^{2}}{e^{y}+1} f(y)$. One of the mean of $y$ is the harmonic mean, $y_{\text {rhms }}=\left\langle y^{-2}\right\rangle^{-1 / 2}=$ $\left(\frac{\Gamma(3) \eta(3)}{\Gamma(1) \eta(1)}\right)^{1 / 2}=1.61286$.

Just as the present Hubble radius $1 / \mathrm{R}_{\mathrm{H}}{ }^{2}=8 \pi \mathrm{G} \rho_{\mathrm{c}} / 3$, we may write neutrino horizon as $1 / \mathrm{R}_{v}{ }^{2}=8 \pi \mathrm{G}_{\mathrm{v}} / 3$. The Jeans radius is given by $\left(\frac{\pi}{R_{J}}\right)^{2}=k_{J}^{2}=\frac{4 \pi G \rho_{v}}{v^{2}}=\frac{3}{2 v^{2} R_{v}^{2}}$.

The Jeans mass that is contained within the Jeans radius, $M_{J}=\frac{4 \pi R_{J}^{3} \rho_{v}}{3}=\frac{1}{2}\left(\frac{R_{J}^{3}}{R_{v}^{2}}\right)$, has the momentum dependence,
$M_{J}=C \frac{x^{2} y^{3}}{\left(x^{2}+y^{2}\right)^{7 / 4}}$, where the constant $C=\frac{15 X 10^{18}}{m_{e v}^{2} \sqrt{g}} M_{\ominus}$.

Now, substituting $\mathrm{y}$ by, $\mathrm{y}_{\text {rhms }}$ and $\mathrm{x}=1.9$ for which the Jeans mass peaked in the plot of $M_{J}$ versus $x$, we calculate $M_{J}$ for different values of $m_{\tilde{o}}$ that we discussed above to obtain following results, assuming $m_{v_{e}}=m_{v_{\mu}}=m_{v_{\tau}}$ and g $=6$ :

\begin{tabular}{|l|l|l|}
\hline $\begin{array}{c}\mathbf{m}_{\mathbf{v}} \\
(\mathbf{e V})\end{array}$ & \multicolumn{1}{|c|}{ Reference / Source } & $\begin{array}{c}\mathbf{M}_{\mathbf{J}} \text { in the units } \\
\text { of 10 } \\
\text { mass }\end{array}$ \\
\hline 400 & Rolar \\
\hline 130 & Reference [6] & $9.3 \times 10^{-5}$ \\
\hline 8 & Reference [8] & $8.8 \times 10^{-4}$ \\
\hline 5 & Reference [9] & $2.3 \times 10^{-1}$ \\
\hline 0.73 & Reference [10], 2dFGRS & $5.9 \times 10^{-1}$ \\
\hline 0.23 & $\begin{array}{l}\text { Reference [11], 1 } 1^{\text {st }} \text { year WMAP, } \\
\text { ACBAR, CBI and 2dFGRS }\end{array}$ & $2.8 \times 10^{1}$ \\
\hline 0.2 & $\begin{array}{l}\text { Reference [12], 5 year WMAP, } \\
\text { BAO \& SN }\end{array}$ & $3.7 \times 10^{2}$ \\
\hline 0.1 & $\begin{array}{l}\text { Reference [13], CMB, LSS \& } \\
\text { SDSS }\end{array}$ & $1.4 \times 10^{3}$ \\
\hline 0.04 & $\begin{array}{l}\text { Reference [14], PLANCK CMB } \\
\text { \& SDSS }\end{array}$ & $9.3 \times 10^{3}$ \\
\hline 0.01 & Reference [15] & $1.5 \times 10^{5}$ \\
\hline
\end{tabular}

It is seen from the table that the Jeans mass of the structures formed is at least of the Supercluster or Great Attractor if the structures are formed only out of neutrinos. If the only neutrinos of the mass $0.2 \mathrm{eV}$ predicted by current 5 year WMAP team clump together to form the large-scale structures, they are tens of thousands times larger than the Superclusters. So, there must be some other components of 'cold dark matter' along with neutrinos in order to form the structures of the smaller sizes. From $x=m_{v} c^{2} / k_{B} T$, if we calculate the temperature for $\mathrm{x}=1.9$ and $\mathrm{m}_{v}=0.2 \mathrm{eV}$, it comes to be $1220 \mathrm{~K}$ which is little less than the temperature of 'recombination epoch'.

In the reference [16], $<\mathrm{M}_{\mathrm{J}}>$ has been calculated by different combinations and averaging of $\rho$, v and other parameters and has been plotted against $\mathrm{x}$. It has peaked to different values at different values of $x$. Fig. -1 shows the variation of the peak values of the average Jeans mass $<\mathrm{M}_{\mathrm{J}}>$ with $\mathrm{x}$.

Peak $\mathrm{M}_{\mathrm{J}}$

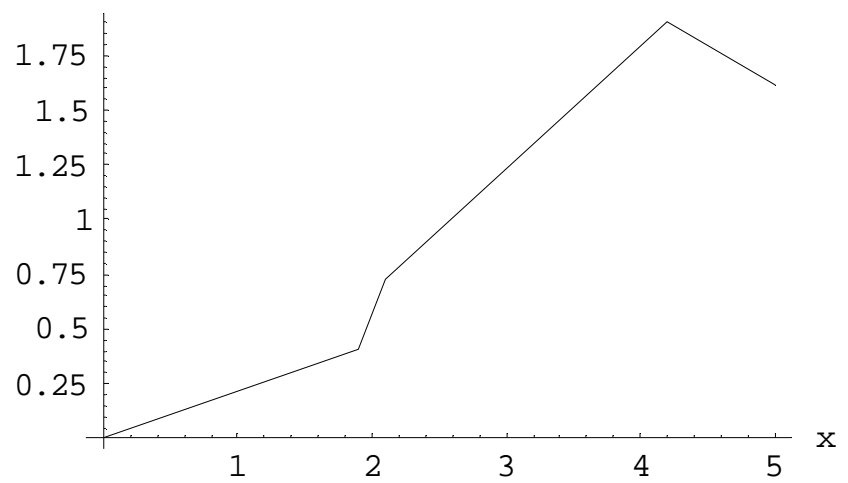

Fig. 1: Plot of peak value of average Jeans mass $\left\langle\mathrm{M}_{\mathrm{J}}\right\rangle$ against the corresponding value of $\mathrm{x}=\mathrm{m}_{\mathrm{v}} \mathrm{c}^{2} / \mathrm{k}_{\mathrm{B}} \mathrm{T}$ for which the peak occurred. 


\section{CONCLUSION:}

From the above analysis, it is seen that if the massive neutrinos are the only candidates to form the large scale structures and they have the mass of present day prediction, then the structures formed are bigger than the Superclusters or Great Attractor and the structures are formed around 'recombination epoch'. For the formation of smaller structures of galactic sizes, there must be other cold dark matter along with neutrinos. We are now looking for the further effects by solving Dirac's equation.

\section{REFERENCES:}

[1] Alpher, R.A., Follin, J.W. \& Hermann, R.C. 1953. Phys. Rev. 92: 1347

[2] Sarkar, S. arXiv:hep-ph/0302175v2.

[3] Zel'dovich, Ya.B. 1965. Adv. Astron. Astrophys. 3: 241.

[4] Chiu, H. 1966. Ann. Rev. Nucl. Sci. 16: 591.

[5] Pontecorvo, B. \& Smorodinski, Ya. 1962. Sov. Phys. JETP. 14: 173.
[6] Gershtein, S.S. \& Zeldovich, Ya.B. 1966. JETP Lett. 4: 120.

[7] Marx, G. \& Szalay, A.S. 1972. Proc. Neutrino'72, Balatonfurd. 1: 123.

[8] Cowlik, R. \& McClelland, J. 1972. Phys. Rev. Lett. 29: 669.

[9] Bernstein, J. \& Geinberg, G. 1981. Phys. Lett. B 101: 39.

[10] Elgaroy, O. et. al. 2002. Phys. Rev. Lett. 89: 061301.

[11] Spergel, D.N. et al. 2003. Astrophys. J. [arXiv:astro-phy/ 0302209].

[12] Komatsu, E. et al. arXiv:astro-ph/0803.0547v1.

[13] Hu, W., Eisenstein, D.J. and Tegmark, M. Phys. Rev. Lett. 80: 5255 .

[14] Hannestad, S. 2003. Phys. Rev. D 67: 085017.

[15] Pastor, S. arXiv:hep-ph/0306233v1.

[16] Dhungel, P.R., Sharma, S.K. \& Khanal, U. 2004. Scientific World, 2: 7. 